\title{
Hepatitis B virus infection and the kidney: a wide range of manifestations
}

Rui Barata ${ }^{1}$, Patrícia Alves ${ }^{1}$, Tiago Assis Pereira ${ }^{1}$, Mário Góis ${ }^{1,2}$, Helena Viana ${ }^{1,2}$, Fernando Nolasco ${ }^{1}$

${ }^{1}$ Nephrology Department, Hospital de Curry Cabral - Centro Hospitalar de Lisboa Central

2 Laboratory of Renal Morphology, Hospital de Curry Cabral - Centro Hospitalar de Lisboa Central

\section{CLINICAL PRESENTATION}

We present a case of a 46-year-old African male referred for chronic kidney disease. His past medical history included poorly controlled hypertension, active smoking and non-treated hepatitis B virus (HBV) infection (viral load of $4114 \mathrm{UI} / \mathrm{mL}$ ), without laboratorial or radiological evidence of chronic liver disease. His family medical history was negative for renal disease.

Renal disfunction had been detected two years earlier (serum creatinine [Scr] of $1.3 \mathrm{mg} / \mathrm{dL}$ at the time) and had been progressively increasing since then (Scr of $2.49 \mathrm{mg} / \mathrm{dL}$ at the time of referral, estimated glomerular filtration rate [eGFR] of $37 \mathrm{~mL} / \mathrm{min} / 1.73 \mathrm{~m}^{2}$ ). Urinalysis was positive for hematuria and proteinuria, with an urine protein to creatinine ratio (PCR) of $1383 \mathrm{mg} / \mathrm{g}$. Physical examination was unremarkable. Albumin concentration was normal. Human immunodeficiency virus (HIV) and hepatitis C virus (HCV) testing were negative. Immunological study was negative, including antinuclear antibody, serum complement components $\mathrm{C} 3$ and $\mathrm{C} 4$, cryoglobulins and anti-phospholipase $\mathrm{A} 2$ receptor antibody. A kidney biopsy was performed.

Figure 1

HE. $\times 200$

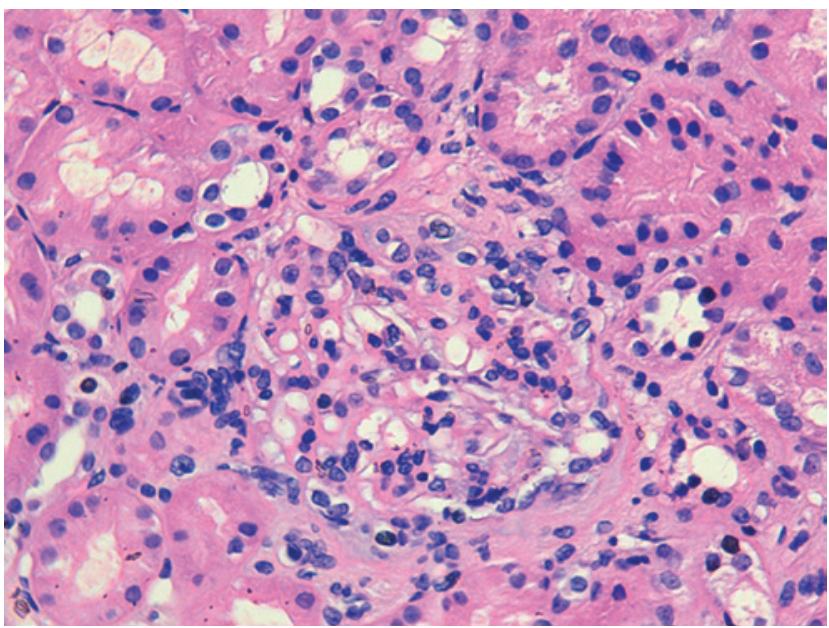

\section{Figure 2}

Methenamine silver. $\times 200$

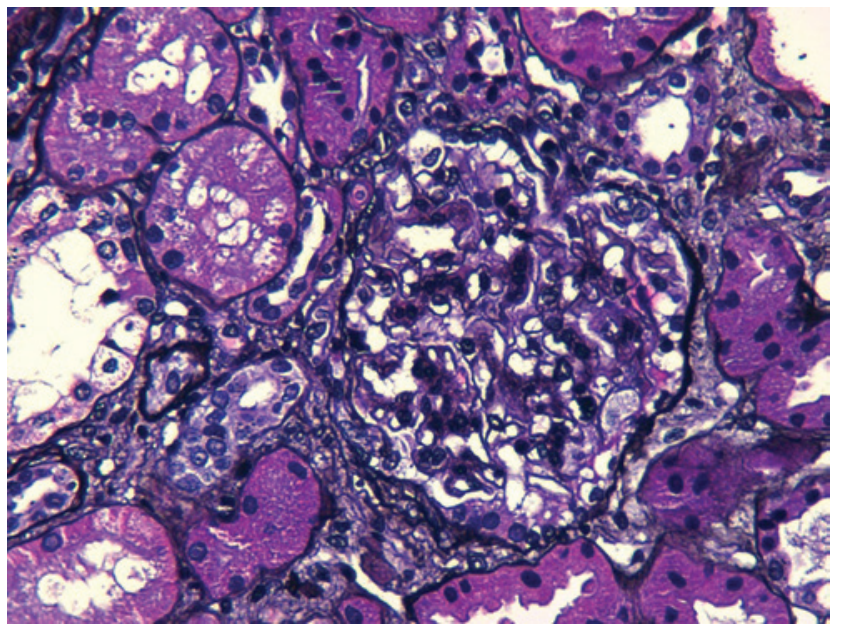

Figure 3

Masson's trichrome. $\times 40$

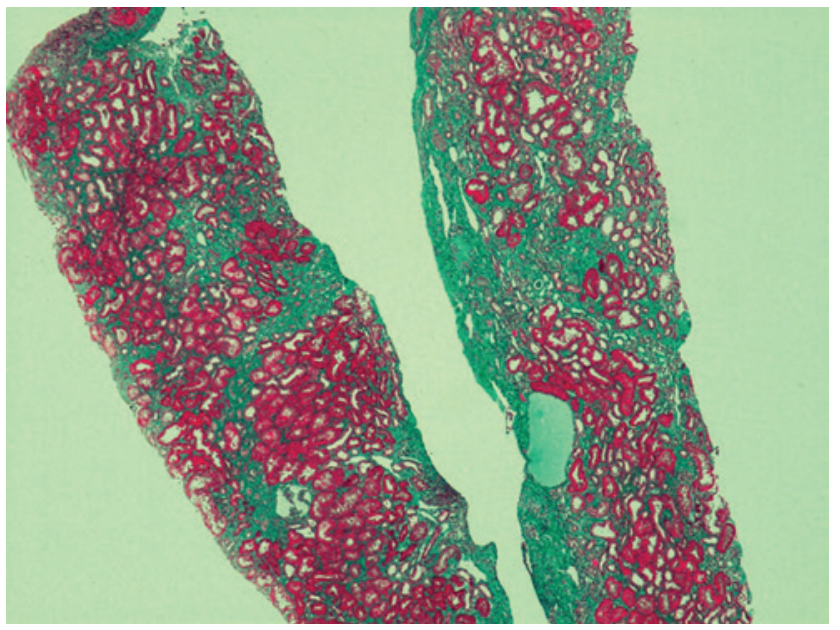




\section{Figure 4}

Immunofluorescence. Anti-IgA. x200

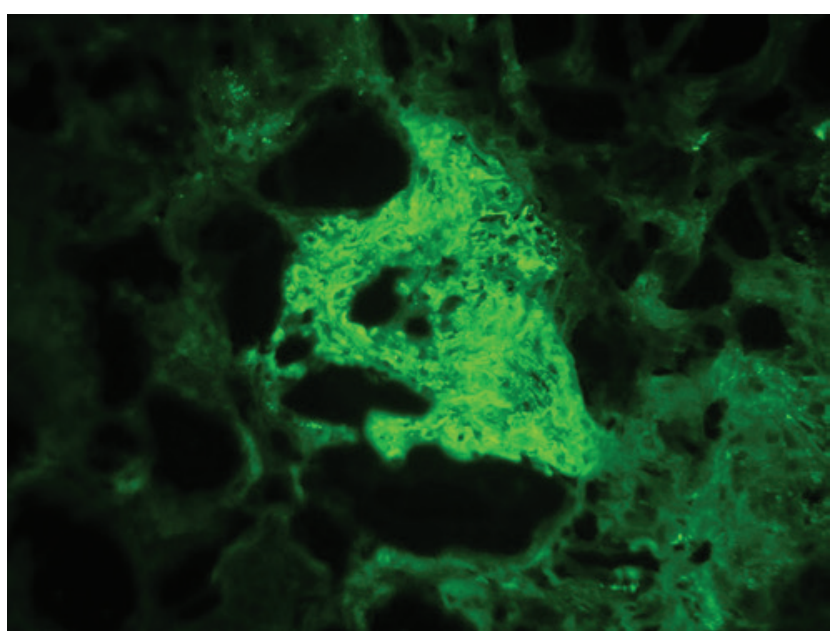

\section{QUESTIONS}

1. What is the most likely diagnosis, given the clinical history and presentation?

2. What is the diagnosis considering light microscopy and immunofluorescence studies?

3. How would you treat this patient?

4. What is our patient's prognosis?

\section{ANSWERS}

1. What is the most likely diagnosis, given the clinical history and presentation?

Given the patient's young age, non-controlled HBV infection and the presence of hematoproteinuria, the most likely diagnosis is glomerulopathy associated with HBV infection.

$\mathrm{HBV}$ is the most common chronic viral infection worldwide, with renal disease occurring in 3-5\% of those patients. Infection with HBV may be associated with a variety of renal diseases, the most common being membranous nephropathy, usually manifested as proteinuria and microscopic hematuria. ${ }^{1}$ However, several other renal manifestations can be present with chronic HBV infection. They can generally be pathophysiology distinguished as those that result from immunecomplex glomerulonephritis (GN), such as membranous nephropathy, membranoproliferative GN (MPGN) and IgA nephropathy; and those associated with immune complex-associated vasculitis, such as polyarteritis nodosa (PAN). ${ }^{2}$

Given the lack of systemic manifestations, progressive renal disfunction with proteinuria and microhematuria, the negative immunological findings, and it being the most frequent renal manifestation in patients with HBV infection, the most probable diagnosis is membranous nephropathy. In addition to that, the hypothesis of hypertensive nephrosclerosis (alone or associated) cannot be excluded. However, the patient's age and the presence of hematuria make this a less probable cause. Furthermore, other causes of glomerulopathy must be considered.

\section{What is the diagnosis considering light microscopy and immuno- fluorescence studies?}

The kidney biopsy contained 4 glomeruli and a large-sized artery. In light microscopy, an ischemic glomerulus was visible, and the remainder had mesangial proliferation (Figure 1), with 2 segmental endocapillary hypercellularity lesions (Figure 1 and 2), and no evidence of extracapillary proliferation. A slight podocyte hypertrophy was detected. Fibrosis involving about $30 \%$ of cortical was also visible (Figure 3).

Immunofluorescence revealed granular mesangial deposits of IgA (Figure 4), C3 and lambda light chains. There was also a segmental deposit of IgM.

The diagnosis is IgA nephropathy (Oxford classification: M1, E1, $\mathrm{S} 1, \mathrm{~T} 1, \mathrm{C} 0)$.

\section{How would you treat this patient?}

Although some of the most frequent renal manifestations of HBV infection are well characterized, such as membranous nephropathy, evidence regarding IgA nephropathy associated with HBV infection, including its treatment, is lacking. ${ }^{3}$

General measures to slow the rate of renal function decline and reduce proteinuria are of most importance in patients with IgA nephropathy. These include angiotensin-converting enzyme (ACE) inhibitors or angiotensin II receptor blockers (ARBs), as well as smoking cessation and weight loss ${ }^{4}$. Hypertension must also be controlled.

Assuming HBV infection as the cause of IgA nephropathy, antiviral therapy must be started. There are some anecdotal cases of resolution of IgA nephropathy in patients with HBV infection treated with proper anti-viral therapy, emphasizing the role of HBV in its pathophysiology ${ }^{1}$. However, a study comparing patients with IgA nephropathy with and without HBV infection found no difference in the decline of renal function, nor protein excretion between the two groups. Moreover, there was no difference in those parameters among patients with and without anti-viral therapy. ${ }^{3}$ Those findings raise the question of whether treatment of $\mathrm{HBV}$ infection without immunosuppression may be sufficient in patients with IgA nephropathy, and about the role of $\mathrm{HBV}$ in this disease.

The use of immunosuppression in this case, namely corticosteroids, is more controversial. Most studies showed higher benefit in patients with proteinuria $>3-3.5 \mathrm{~g} / \mathrm{d} .{ }^{4}$ In this case, the patient had worsening kidney function, proteinuria and hypertension, clinical findings that added to histological signs of renal ongoing inflammatory process, which would support the use of immunosuppressive medication. 
Further, the patient's young age and few comorbidities make him a good candidate for immunosuppression. Thus, a reasonable approach would be to start general measures, and evaluate their impact on renal function and proteinuria in 3-6 months.

In our patient, we started nephroprotective measures, control of hypertension and, in conjunction with the infectious diseases department, treatment of HBV infection with entecavir. Despite this, after normalization of blood pressure and undetectable HBV viral load, there was no notable change in renal function or proteinuria. Hence, we decided to start a 6-month course of immunosuppression with prednisolone (1 $\mathrm{mg} / \mathrm{kg}$ per day).

\section{What is our patient's prognosis?}

Our patient has several clinical markers of poor prognosis, including renal impairment and proteinuria, and histopathological findings, with mesangial hypercellularity, endocapillary proliferation, segmental glomerulosclerosis. In addition to that, the coexistence of hypertension and ongoing smoking are also associated with poor outcomes.
Race may also be an important prognostic factor. ${ }^{4}$ Moreover, the lack of response after hypertension and HBV infection may also predict a poor prognosis.

Disclosure of potential conflicts of interest: none declared

\section{References}

1. Gupta A, Quigg RJ. Glomerular diseases associated with Hepatitis B and C. Adv Chronic Kidney Dis. 2015 Sep;22(5):343-351.

2. Kupin WL. Viral-Associated GN: Hepatitis B and other viral infections. Clin J Am Soc Nephrol. 2017 Sep 7;12(9):1529-1533.

3. Sun IO, Hong YA, Park HS, Choi SR, Chung BH, Park CW, Yang CW, Kim YS, Choi BS. Clinical characteristics and treatment of patients with IgA nephropathy and Hepatitis B surface antigen. Ren Fail. 2013;35(4):446-451.

4. Rodrigues JC, Haas M, Reich HN. IgA nephropathy. Clin J Am Soc Nephrol. 2017 Apr 3;12(4):677-686.

\section{Correspondence to:}

Rui Barata, MD

Nephrology Department, Hospital de Curry Cabral - Centro Hospitalar de Lisboa Central

E-mail: rui.f.barata@gmail.com 